\title{
A importância do planejamento urbano nas cidades médias brasileiras $^{1}$
}

\section{The importance of urban planning in Brazilian medium size cities}

\author{
Verona, Tainá F. ${ }^{1}$; Barbosa, Gisele S. ${ }^{2}$ \\ 1 Universidade Federal do Rio de Janeiro, Escola Politécnica, Programa de \\ Engenharia Urbana, Av. Athos da Silveira Ramos, 149, CT, bloco D, sala D101, \\ CEP 21941-909, Brasil, taina.verona@poli.ufrj.br \\ 2 Universidade Federal do Rio de Janeiro, Escola Politécnica, Departamento \\ de Expressão Gráfica, Programa de Engenharia Urbana, \\ giselebarbosa@poli.ufrj.br
}

\begin{abstract}
RESUMO
O Estatuto das Cidades regulamentou muitos instrumentos importantes para garantia do direito à cidade igualitário e da função social da moradia. Porém todos esses instrumentos devem ser aplicados por cada município, de acordo com o que é previsto em seu plano diretor. Ao longo das últimas décadas o número de cidades médias no país tem aumentado e seu destaque dentro das redes urbanas em que estão inseridas vem crescendo. O presente estudo é uma discussão teórica, parte dos resultados discursivos iniciais de uma pesquisa de mestrado, acerca de como a questão do planejamento urbano é tratado nas cidades médias. Com relação aos procedimentos técnicos e metodológicos a pesquisa classifica-se como uma pesquisa bibliográfica. Através de uma revisão, conclui-se que apesar dos mecanismos presentes na legislação ainda há um longo caminho a percorrer para uma gestão urbana mais inclusiva. Nas cidades médias, devido a menor capacidade técnica dos órgãos gestores, existe uma dificuldade maior em aplicar os instrumentos previstos nos planos diretores. O recorte da análise do planejamento urbano em cidades entre 100 e 500 mil habitantes é importante para entender as especificidades da governança urbana nesses espaços.
\end{abstract}

Palavras-chave: planejamento urbano, cidades médias, plano diretor.

\begin{abstract}
The Statute of Cities regulated many important instruments to guarantee the right to equal access to the city and the social function of habitation. However, all these instruments must be applied by each municipality, according to what is predict in its master plan. Over the last decades the number of medium-sized cities in the country has increased and its prominence within the urban networks has been growing. The present study is a theoretical discussion, part of the initial discursive results of a master's degree research, about how the issue of urban planning is addressed in medium-sized cities. With respect to technical and methodological

\footnotetext{
${ }^{1}$ VERONA, Taina F.; BARBOSA, Gisele S. A importância do planejamento urbano nas cidades médias brasileiras. In: II SIMPÓSIO NACIONAL DE GESTÃo E ENGENHARIA URBANA: SINGEURB, 2019, São Paulo. Anais... Porto Alegre: ANTAC, 2019.
} 
procedures, the research is classified as a bibliographical research. Through a review, it is concluded that despite the mechanisms presented in the legislation, there is still a long way to go for a more inclusive urban management. In medium-sized cities, due to the insufficient technical capacity of the municipal managers, there is a greater difficulty in applying the instruments of the master plans. To focus the analysis of the urban planning in cities between 100 and 500 thousand inhabitants is important to understand the particularity of the governance of these spaces.

Keywords: urban planning, medium-sized cities, master plan.

\section{INTRODUÇÃO}

A preocupação com o planejamento urbano existe no país desde o início do século XX, mas somente na Constituição Federal de 1988 que se iniciou a institucionalização do direito à cidade e da função social da moradia. Em 2001 foi aprovado o Estatuto das Cidades que regulamentou os artigos 182 e 183 da Constituição e criou ferramentas para que as administrações municipais promovessem o desenvolvimento urbano buscando equidade de acesso à cidade e sustentabilidade.

Porém, nas cidades médias e pequenas a situação, muitas vezes, é mais complicada. Seja pela falta de recursos técnicos e financeiros ou por falta de interesse das autoridades locais, existem cidades em que o planejamento urbano não leva em consideração as particularidades locais e tudo se resume a um plano diretor com diretrizes genéricas e pouca aplicação prática.

Através de uma pesquisa bibliográfica, fez-se uma discussão teórica sobre as cidades médias no Brasil e sobre o planejamento urbano nesses locais. Buscando entender as especificidades da urbanização das cidades médias e examinar as dificuldades apresentadas na gestão urbana.

\section{PLANEJAMENTO URBANO E CIDADES MÉDIAS}

\subsection{Planejamento urbano no Brasil}

A partir da Constituição Federal (CF) de 1988 o papel do plano diretor como principal meio do planejamento urbano municipal foi consolidado no país, uma vez que o artigo 182 da Constituição requer que toda cidade com mais de 20 mil habitantes elaborasse esse documento?2.

A lei 10.257/2001, Estatuto das Cidades (EC), define diretrizes para a administração municipal com relação ao planejamento e gestão da cidade e regulamenta conjuntos de instrumentos que possuem o intuito de concretizar as políticas urbanas municipais. Entre eles estão a instituição de zonas especiais de interesse social (ZEIS); parcelamento compulsório; direito de preempção; outorga onerosa; transferência do direito de construir e as operações urbanas consorciadas.

Fica a cargo de cada município a regulamentação dos instrumentos conforme as estratégias de desenvolvimento urbano. O que é previsto no EC não soluciona os problemas, a transformação urbana depende da aplicação dos instrumentos de maneira coordenada conforme estratégia de desenvolvimento explícita no Plano Diretor (PD) (BARROS et al, 2010).

\footnotetext{
2 "Art. 41. O plano diretor é obrigatório para cidades: I - com mais de vinte mil habitantes; II - integrantes de regiões metropolitanas e aglomerações urbanas; III - onde o Poder Público municipal pretenda utilizar os instrumentos previstos no $\S 4$ ㅇ do art. 182 da Constituição Federal; IV - integrantes de áreas de especial interesse turístico; V - inseridas na área de influência de empreendimentos ou atividades com significativo impacto ambiental de âmbito regional ou nacional. VI Incluídas no cadastro nacional de Municípios com áreas suscetíveis à ocorrência de deslizamentos de grande impacto, inundações bruscas ou processos geológicos ou hidrológicos correlatos" (Estatuto da Cidade - Lei 10257/01).
} 


\subsection{Cidades médias}

Existe uma dificuldade de fixar o conceito de cidade média em todo o mundo, mas em todas as discussões reconhece-se que essa concepção vai muito além do tamanho da população.

Tomar a população urbana como proxy do tamanho do mercado local e indicador do nível de infraestrutura existente e grau de concentração das atividades pode ser útil para uma primeira aproximação (AMORIM E SERRA, 2001).

Aspectos como relações externas, estrutura interna e problemas sociais variam bastante entre países e mesmo entre regiões do mesmo país. Desse modo a classificação como cidade média depende do nível de desenvolvimento alcançado, da posição e das condições geográficas e do processo de formação histórico-social de cada local (AMORIM E SERRA, 2001).

Em suma, estudiosos concordam que cidades médias servem como centro de serviços e equipamentos tanto para os habitantes do mesmo núcleo urbano como aqueles que residem em sua área de influência. Interagindo com amplas áreas territoriais e desenvolvendo funções de distribuição e intermediação, sendo dotadas de uma série de infraestruturas coletivas (OLIVEIRA JR, 2008).

\subsubsection{Cidades médias no Brasil}

Após a década de 1970 no Brasil, emergiram novos centros de decisões governamentais, de produção industrial e pontos de convergência e geração de fluxos de pessoas, mercadorias e informações. E as cidades médias se tornaram fortes e importantes centralidades em âmbito regional (FRANÇA E SOARES, 2012).

As grandes metrópoles viram sua participação relativa na população total do país estagnar e as cidades médias passaram a ganhar mais espaço na demografia brasileira, o que vem sendo chamado de desmetropolização (FRANÇA E SOARES, 2012). Esse processo pode ser observado na Tabela 1.

Tabela 1 -Número de municípios e população nos censos demográficos de 1970 a 2010

\begin{tabular}{|c|c|c|c|c|c|c|c|c|c|}
\hline \multirow{3}{*}{$\begin{array}{c}\text { Classes de } \\
\text { população dos } \\
\text { municípios (X } \\
1000 \\
\text { habitantes) }\end{array}$} & \multicolumn{9}{|c|}{ Número de municípios e população nos Censos Demográficos } \\
\hline & \multicolumn{3}{|c|}{01.09 .1970} & \multicolumn{3}{|c|}{01.09 .1991} & \multicolumn{3}{|c|}{01.08 .2010} \\
\hline & Munic & $\begin{array}{c}\text { Pop } \\
\text { (X1000) }\end{array}$ & $\%$ Pop & Munis & $\begin{array}{l}\text { Pop } \\
\text { (X1000) }\end{array}$ & \% Pop & Munic & $\begin{array}{c}\text { Pop } \\
\text { (X1000) }\end{array}$ & $\%$ Pop \\
\hline Total & 3952 & 94508 & $100 \%$ & 4491 & 146917 & $100 \%$ & 5565 & 190755 & $100 \%$ \\
\hline Até 2 & 56 & 92 & $0,10 \%$ & 58 & 91 & $0,06 \%$ & 118 & 197 & $0,10 \%$ \\
\hline De 2 a 100 & 3802 & 61823 & $65,42 \%$ & 4246 & 75975 & $51,71 \%$ & 5164 & 86121 & $45,15 \%$ \\
\hline De 100 a 500 & 83 & 14610 & $15,46 \%$ & 162 & 32073 & $21,83 \%$ & 245 & 48565 & $25,46 \%$ \\
\hline Mais de 500 & 11 & 17982 & $19,03 \%$ & 25 & 38776 & $26,39 \%$ & 38 & 55871 & $29,29 \%$ \\
\hline
\end{tabular}

Fonte: IBGE, 2010 (tabela adaptada pelos autores)

As cidades em regiões metropolitanas ou polarizadas por capitais de estados, em geral, não entram na classificação de cidades médias, pois não possuem condições necessárias para polarizar e exercer funções de centralidade (OLIVEIRA JR., 2008). Porém como o ritmo mais elevado de crescimento do conjunto de cidades médias inclui as metropolitanas, não conta exatamente como um processo de desconcentração populacional, como aponta Carvalho (2003). 


\section{DISCUSSÃO E RESULTADOS}

\subsection{Planejamento urbano nas cidades médias}

De acordo com dados da Pesquisa de Informações Básicas Municipais de 2015 - MUNIC, realizada pelo IBGE, 100\% das cidades entre 100 e 500 mil habitantes já possui a lei do Plano Diretor aprovada. Porém, alguns dos instrumentos de planejamento existentes no Estatuto das Cidades não são aplicados em um número considerável de municípios.

Conforme pode ser visto na Tabela 2, alguns instrumentos como ZEIS, estudo de impacto de vizinhança, zoneamento e outorga onerosa já aparecem na legislação urbana da maior parte das cidades médias. Mas outros como servidão administrativa, direito de superfície e concessão para fins de moradia estão presentes em menos de metade dos municípios (IBGE, 2015).

Tabela 2 -Municípios que possuem instrumentos de planejamento em sua legislação urbanística

\begin{tabular}{|c|c|c|c|c|c|c|c|c|c|c|}
\hline Pop (X1000) & A & B & C & D & $\mathbf{E}$ & $\mathbf{F}$ & $\mathbf{G}$ & $\mathbf{H}$ & I & $J$ \\
\hline Brasil & $51 \%$ & $42 \%$ & $88 \%$ & $61 \%$ & $59 \%$ & $35 \%$ & $53 \%$ & $25 \%$ & $34 \%$ & $66 \%$ \\
\hline Até 5 & $34 \%$ & $25 \%$ & $90 \%$ & $49 \%$ & $44 \%$ & $19 \%$ & $47 \%$ & $12 \%$ & $17 \%$ & $50 \%$ \\
\hline De 5 a 10 & $37 \%$ & $28 \%$ & $85 \%$ & $48 \%$ & $46 \%$ & $22 \%$ & $44 \%$ & $14 \%$ & $20 \%$ & $55 \%$ \\
\hline De 10 a 20 & $40 \%$ & $32 \%$ & $82 \%$ & $54 \%$ & $49 \%$ & $28 \%$ & $49 \%$ & $18 \%$ & $25 \%$ & $65 \%$ \\
\hline De 20 a 50 & $72 \%$ & $66 \%$ & $91 \%$ & $79 \%$ & $79 \%$ & $51 \%$ & $63 \%$ & $40 \%$ & $54 \%$ & $81 \%$ \\
\hline De 50 a 100 & $90 \%$ & $82 \%$ & $97 \%$ & $94 \%$ & $95 \%$ & $69 \%$ & $72 \%$ & $54 \%$ & $71 \%$ & $93 \%$ \\
\hline De 100 a 500 & $97 \%$ & $91 \%$ & $98 \%$ & $98 \%$ & $99 \%$ & $81 \%$ & $77 \%$ & $69 \%$ & $87 \%$ & $92 \%$ \\
\hline Mais de 500 & $100 \%$ & $98 \%$ & $95 \%$ & $100 \%$ & $100 \%$ & $93 \%$ & $78 \%$ & $83 \%$ & $95 \%$ & $100 \%$ \\
\hline Pop (X1000) & $K$ & $\mathbf{L}$ & $M$ & $\mathbf{N}$ & 0 & $\mathbf{P}$ & $\mathbf{Q}$ & $\mathbf{R}$ & $S$ & $\mathbf{T}$ \\
\hline Brasil & $28 \%$ & $13 \%$ & $24 \%$ & $21 \%$ & $23 \%$ & $12 \%$ & $17 \%$ & $26 \%$ & $13 \%$ & $25 \%$ \\
\hline Até 5 & $16 \%$ & $8 \%$ & $14 \%$ & $11 \%$ & $14 \%$ & $6 \%$ & $7 \%$ & $14 \%$ & $6 \%$ & $13 \%$ \\
\hline De 5 al0 & $17 \%$ & $10 \%$ & $18 \%$ & $13 \%$ & $15 \%$ & $7 \%$ & $9 \%$ & $18 \%$ & $9 \%$ & $15 \%$ \\
\hline De10 a20 & $20 \%$ & $12 \%$ & $18 \%$ & $17 \%$ & $19 \%$ & $8 \%$ & $11 \%$ & $20 \%$ & $10 \%$ & $19 \%$ \\
\hline De 20 a 50 & $45 \%$ & $20 \%$ & $34 \%$ & $32 \%$ & $35 \%$ & $20 \%$ & $27 \%$ & $39 \%$ & $21 \%$ & $40 \%$ \\
\hline De 50 a 100 & $59 \%$ & $22 \%$ & $41 \%$ & $41 \%$ & $42 \%$ & $25 \%$ & $41 \%$ & $45 \%$ & $23 \%$ & $52 \%$ \\
\hline De 100 a 500 & $65 \%$ & $26 \%$ & $58 \%$ & $52 \%$ & $47 \%$ & $30 \%$ & $48 \%$ & $65 \%$ & $23 \%$ & $62 \%$ \\
\hline Mais de 500 & $49 \%$ & $27 \%$ & $78 \%$ & $68 \%$ & $66 \%$ & $29 \%$ & $54 \%$ & $80 \%$ & $27 \%$ & $71 \%$ \\
\hline
\end{tabular}

Legenda: A - Área e/ou zona especial de interesse social; B - Área e/ou zona especial de interesse; C- Perímetro urbano; D- Parcelamento do solo; E -Zoneamento ou uso e ocupação do solo; F- Solo criado ou outorga onerosa do direito de construir; G- Contribuição de melhoria; H- Operação urbana consorciada; I - Estudo de impacto de vizinhança; J- Código de obras; K -Zoneamento ambiental ou zoneamento ecológico-econômico; L- Servidão administrativa; M- Tombamento; N- Unidade de Conservação; O - Concessão de uso especial para fins de moradia; P- Usucapião especial de imóvel urbano; Q- Direito de superfície; R- Regularização fundiária; S- Legitimação de posse; T -Estudo prévio de impacto ambiental

Fonte: MUNIC - IBGE, 2015 (tabela adaptada pelos autores)

Apesar da incorporação desses instrumentos de planejamento nos planos diretores, estudos qualitativos mostraram que, em grande parte das vezes, a falta de estratégias de implantação vinculadas à realidade local de cada cidade dificulta que os objetivos de uma gestão urbana mais inclusiva sejam alcançados.

Conforme aponta Rolnik (2012), apesar da quantidade de planos diretores com presença de instrumentos como as ZEIS, o emprego deles ou a vinculação com estratégias de desenvolvimento urbano são deficientes. Além disso, os mecanismos de participação popular previstos na lei ainda não são eficientes para romper com as velhas relações políticas. De modo que as reivindicações da população carente raramente são ouvidas no momento das decisões.

Oliveira (2011), ao analisar a eficácia da ZEIS, pontua que apesar da grande maioria dos planos prever a instituição dessas zonas nem sempre há delimitação e poucos deles as regulamentam ou dão orientações para a sua regulamentação posterior. Segundo ele, a 
definição de ZEIS raramente se relaciona com estratégias voltadas para a promoção da ocupação das áreas já urbanizadas pela população de baixa renda. Na verdade, em geral, os planos delimitam áreas já ocupadas por assentamentos precários para fins de regularização fundiária e urbanística.

Segundo Costa (2016), alguns dos motivos para o uso não efetivo dos instrumentos do Estatuto são: instrumentos previstos no PD não são auto aplicáveis e exigem regulamentações posteriores; planos diretores que trazem uma réplica do portfólio de instrumentos sugeridos pelo EC, sem aderência à realidade dos municípios; e falta de articulação entre os dispositivos do PD e o planejamento orçamentário.

Sposito (2006) observa que há uma tendência à idealização e proposição de políticas urbanas orientadas pela associação entre urbanização e modernização, e da oposição entre cidade e natureza. Essa tendência, presente no Brasil em cidades de diferentes portes e importâncias, é acentuada nas cidades médias. Assim, como aponta Carvalho (2003), a urbanização nesses locais passa a reproduzir, em outra escala, os mesmos problemas das metrópoles. Para além dessa reprodução, o crescimento das cidades médias atesta a tendência dessa nova urbanização em tornar indiferenciados os espaços metropolitanos e das cidades médias.

\section{CONCLUSÕES}

As cidades médias estão se urbanizando de maneira semelhante às grandes metrópoles. Mesmo que a legislação federal regulamente diversos mecanismos importantes para a garantia do direito à cidade para todos, da função social da habitação e do desenvolvimento sustentável, ainda existem obstáculos para que sejam aplicados da maneira ideal.

A existência dos instrumentos nos planos diretores não assegura uma gestão urbana com mais equidade, seja nas cidades grandes ou pequenas. Mas nas cidades menores, a incapacidade técnica e institucional, a descontinuidade das gestões municipais e a força política de interesses particulares, como a especulação imobiliária, tem um peso maior na governança. Portanto, a implementação das diretrizes de planejamento urbano previstas no PD se torna mais complexa.

Enfim, a teoria e a prática do planejamento e da gestão urbana ainda são muito distantes uma da outra. O que causa uma descrença popular com relação à metodologia de planejamento urbano e ao poder da participação nos processos decisórios e, consequentemente, diminui ainda mais a eficácia dos instrumentos participativos. O plano diretor é um instrumento importante para os municípios menores, com menos recursos a serem aplicados no desenvolvimento urbano, mas ainda precisa ser melhor aplicado pela administração pública.

\section{REFERÊNCIAS}

AMORIM, O., SERRA, R. V. Evolução e perspectivas do papel das cidades médias no planejamento urbano e regional. Cidades médias brasileiras. Rio de Janeiro: IPEA, 2001

BRASIL. Constituição da República Federativa do Brasil de 1988. Brasília, DF. Disponível em: <http://www.planalto.gov.br/ccivil_03/constituicao/constituicao.htm>. Acesso em: abril de 2018.

BRASIL. Estatuto da Cidade - Lei n. 10257, de 10 de julho de 2001. Regulamenta os arts. 182 e 183 da Constituição Federal, estabelece diretrizes gerais da política urbana e dá outras providências. Diário Oficial da União. Brasília, DF, 10 jul. 2001. Disponível em: <http://www.planalto.gov.br/ccivil_03/leis/leis_2001/110257.htm>. Acesso em: junho de 2018 
BARROS, A. M. F. B., CARVALHO, C. S. e MONTANDON, D. T. O Estatuto da Cidade comentado. In: CARVALHO, Celso Santos e ROSSBACH, Anaclaudia (org). O Estatuto da Cidade : comentado/ The City Statute of Brazil : a commentary. - São Paulo:Ministério das Cidades: Aliança das Cidades, 2010 Disponível em:

<http://www.cidades.gov.br/images/stories/ArquivosSNPU/Biblioteca/PlanelamentoUrbano/E statutoComentado_Portugues.pdf> Acesso em: junho de 2018.

CARVALHO, E. Exclusão social e crescimento das cidades médias brasileiras. Scripta Nova. Revista electrónica de geografía y ciencias sociales, 2003

COSTA, M. A.(Org)O Estatuto da Cidade e a Habitat III: um balanço de quinze anos da política urbana no Brasil e a Nova Agenda Urbana. 2016. Disponível em:

<http://www.ipea.gov.br/portal/index.php?option=com_content\&view=article\&id=28583> Acesso em: agosto de 2018.

FRANÇA, I.S.; SOARES, B. R. Rede urbana regional, cidades médias e centralidades: estudo de Montes Claros e dos centros emergentes de Pirapora, Janaúba e Januária no Norte de Minas Gerais. In:Revista Brasileira de Estudos Urbanos e Regionais,Recife, v.14, n. 2, p, 169$185,2012$.

IBGE INSTITUTO BRASILEIRO DE GEOGRAFIA E ESTATÍSTICA. Dados do censo populacional, 2010. Disponível em: < https://censo2010.ibge.gov.br/resultados.html > Acesso em: junho de 2018.

Pesquisa de informações básicas municipais: Perfil dos Municípios Brasileiros 2015. Rio de Janeiro: IBGE, 2016. Disponível em:

<https://biblioteca.ibge.gov.br/visualizacao/livros/liv95942.pdf>. Acesso em: abril de 2019.

OLIVEIRA, F. L. Os Novos Planos Diretores Municipais Brasileiros: uma avaliação preliminar. Anais do XIV Encontro Nacional da ANPUR. Rio de Janeiro: ANPUR, 2011.

OLIVEIRA JR., G. A. Redefinição da centralidade urbana em cidades médias. Sociedade \& Natureza,Uberlândia, v. 20, n. 1, 2008.

ROLNIK, R.; RIBEIRO, A. C. T.; VAZ, L. F.; SILVA, M. L. P. "10 Anos do Estatuto da Cidade: Das Lutas pela Reforma Urbana às Cidades da Copa do Mundo". In: RIBEIRO, A.C.T; VAZ, L.F.; SILVA,M.L.P. (Org.). Quem planeja o território? Atores, arenas e estratégias. 1 ed. Rio de Janeiro: Letra Capital/ANPUR, 2012.

SPOSITO, Maria Encarnação Beltrão. O desafio metodológico da abordagem interescalar no estudo de cidades médias no mundo contemporâneo. Revista Cidades, vol.3, n. 5.

p.143.157. 2006 